\title{
A fusion methodology based on Dempster-Shafer evidence theory for two biometric applications
}

\author{
M. Arif \\ KRL, GPO Box 502 \\ Rawalpindi, Pakistan \\ muhammad.arif@etu.univ-tours.fr
}

\author{
T. Brouard \\ LI, Université François-Rabelais \\ 64 avenue Jean Portalis, \\ 37200 Tours, France
}

\author{
N. Vincent \\ CRIP5-SIP, Université René Descartes \\ 45, rue des Saints Pères, 75270 Paris, France \\ nicole.vincent@math-info.univ-paris5.fr
}

\begin{abstract}
Different features carry more or less rich and varied pieces of information to characterize a pattern. The fusion of these different sources of information can provide an opportunity to develop more efficient biometric system compared when using a feature vector. Thus a new automatic fusion methodology using different sources of information (different feature sets) is presented here. Dempster-Shafer evidence theory is employed for this purpose. For performance evaluation significqntly large data sets of the biometric sources signature and hand shqpe are used. The results on combining different feature vectors compared to a single vector with our approach prove the importance of a fusion process.
\end{abstract}

\section{Introduction}

Fusion is a more and more used step in any process. It aims at improving efficiency of systems. Many methods have been proposed that rely on many different tools. They must be compared not only according to their efficiency but also with respect to the need of a training phase. This is somewhat important when large databases are not available. We have chosen to illustrate our method in the domain of biometrics. Identification and verification are very important matters in access control systems. Various biometric techniques are possible. Here, we are considering hand geometry and handwritten signatures to highlight improvement brought by data fusion. The main concept is to use the complementary nature of several feature sets to increase performances. Our contribution belongs to this trend of trials where different methods such as neural network [1], [2] Bayesian network [3], Hidden Markov models [4], and voting method [5] have been used.

When dealing with a set of features, leading to a representation vectors, different subsets can be considered. In order to make information as complementary as possible we show most efficient is to gather features of the same type and then have a fusion step rather than using the information as a whole. Then we develop an identification/verification system by combining these classifiers based on efficient Dempster-Shafer evidence theory. This theory has been already employed in different application areas such as remote sensing, classifiers combination, environment monitoring, image processing, pattern recognition, etc. Nevertheless, Dempster-Shafer theory's efficiency considerably depends on the function employed as allocation function of mass of belief. In the majority of the cases modeling of the belief function used is empirically fixed by the expert. We propose an automatic assigment of the belief.

First, we describe in section 2 Dempster-Shafer theory and our fusion strategy. We will then specify the features we have chosen in sections 3 and 4 of two biometric applications. In section 5, results are shown.

\section{Dempster-Shafer evidence theory}

In order to combine information coming from different sources, Shafer [6] has created the evidence theory on the bases formulated by Dempster. Evidence is combined in a consistent manner to come to a more complete assessment of what the entire body of evidence implies. Once the belief functions are obtained, fusion is carried out by Dempster's orthogonal combination rule. In this theory, let $\Omega=$ $\left\{\mathrm{H}_{1}, \mathrm{H}_{2}, \ldots, \mathrm{H}_{\mathrm{M}}\right\}$ be the set of possible hypotheses, called the frame of discernment. Let $2^{\Omega}$ denotes the set of the $2^{\mathrm{M}}$ propositions $H$ of $\Omega$. Information bringing an opinion on the state of a system is characterized by a function or a degree of belief $m$. This function is defined by $m: 2^{\Omega} \rightarrow[0,1]$, and has the properties that $m(\phi)=0$ and $\sum_{H \subseteq \Omega} m(H)=1$. A situation of total ignorance is given by $\mathrm{m}(\Omega)=1$ and of total certainty by $m\left(H_{n}\right)=1$ where $H_{n}$ represents a singleton proposition. The total belief committed to $H$, is $\sum_{H^{\prime} \subset H} m\left(H^{\prime}\right)$. 
Now, if we have two information sources $S_{1}$ and $\mathrm{S}_{2}$ with their belief functions $\mathrm{m}_{1}$ and $\mathrm{m}_{2}$, then Dempster's orthogonal operator $\left(m=\oplus_{\mathrm{j}=1}^{\mathrm{J}} \quad \mathrm{m}_{\mathrm{j}}\right)$ is defined by $(\forall H \subseteq \Omega, H \neq \phi)$ :

$\mathrm{m}(\mathrm{H})=\frac{1}{1-K} \sum_{\mathrm{A} \cap \mathrm{B}=\mathrm{H}} \mathrm{m}_{1}(\mathrm{~A}) \cdot \mathrm{m}_{2}(\mathrm{~B})$

where $\mathrm{K}=\sum_{\mathrm{A} \cap \mathrm{B}=\phi} \quad \mathrm{m}_{1}(\mathrm{~A}) \cdot \mathrm{m}_{2}(\mathrm{~B})$

The normalization coefficient $\mathrm{K}$ represents the conflict between two sources.

Let $\mathrm{x}_{\mathrm{j}}(\mathrm{j}=1$ to $\mathrm{N})$ be a collection of reference samples and $C=\left\{\mathrm{C}_{1}, \ldots, \mathrm{C}_{\mathrm{M}}\right\}$ a set of $\mathrm{M}$ classes. Let $\mathrm{x}$ be an incoming sample. Classifying $\mathrm{x}$ means assigning it to one class in $C$, e.g. deciding among a set of $\mathrm{c}$ hypotheses: $\mathrm{x} \in \mathrm{C}_{\mathrm{q}}, \mathrm{q}=1, \ldots, \mathrm{M}$. Using the vocabulary of D-S theory, $C$ can be called the frame of discernment. The focal elements are sets of classes of neighborhing prototypes of $\mathrm{x}$. For modeling the belief function of focal elements, we are introducing a membership function associated with prototypes noted $\mathrm{F}_{\mathrm{xi}}(\mathrm{x})$. The function has values between 0 and 1 . The main variables we have taken into account for a given classifier are (1) Distances $\mathrm{d}\left(\mathrm{x}, \mathrm{x}_{\mathrm{i}}\right)$. normalized with respect to the $\mathrm{k}^{\text {th }}$ prototype's distance value. (2) Rank $\mathrm{R}_{\mathrm{x}}\left(\mathrm{x}_{\mathrm{i}}\right)$ of the prototypes according to their distance values (3) $\mathrm{N}_{\mathrm{x}}(\mathrm{C})$, the number of prototypes belonging to the same class in $\mathrm{k}-\mathrm{NN}$. (4) $\mathrm{V}_{\mathrm{xi}}(\mathrm{x})$, the ratio between the distance $d\left(x, x_{r}\right)$ of current prototype $r$ and sum of all the preceding distances upto the current one. Here is our prototype-based formalism [7]:

$$
\begin{gathered}
F_{x i}(x)=f_{x i}(x) / \sum_{j=1}^{k} f_{x j}(x) \\
f_{x i}(x)=\frac{a_{i}}{1+a_{i}} \\
a_{i}=N_{x}(C) \cdot \sqrt{R_{x}\left(x_{i}\right)} V_{x i}(x) / d\left(x, x_{i}\right)
\end{gathered}
$$

Once $\mathrm{k}$ values of $\mathrm{F}_{\mathrm{xi}}(\mathrm{x})$ are calculated with respect to k-Nearest Neighbors, the neighbors are re-ordered according to these values. These values are then employed to define the belief functions:

$$
\begin{gathered}
m_{i}\left(\left\{\mathrm{H}_{1}\right\}\right)=\mathrm{F}_{\mathrm{x} \sigma(1)}(\mathrm{x}), \quad \ldots, \\
m_{i}\left(\left\{\mathrm{H}_{1}, \mathrm{H}_{2}, \ldots, \mathrm{H}_{\mathrm{g}}\right\}\right)=\mathrm{F}_{\mathrm{x} \sigma(\mathrm{g})}(\mathrm{x})
\end{gathered}
$$

Where index of hypothesis $H$ represents its rank level and $\mathrm{x}_{\sigma(j)}(\mathrm{j}=1, . . \mathrm{k}$ and $\mathrm{g} \leq \mathrm{k})$ represents new ordered prototypes in the neighboring prototypes. The index $g$ represents the rank level of the last class non abundant among the classes of k-NN. A normalisation is finally carried out to respect the constraint of masses of focal elements. In a verification problem, we have only two possible focal elements $\left\{\mathrm{C}_{1}\right\}$ or $\left\{\mathrm{C}_{2}\right\}$, and $\left\{\mathrm{C}_{1}, \mathrm{C}_{2}\right\}$.

Our classification methodology rests on k-NearestNeighbor (k-NN) classifiers principle. Features are divided into various separate sets and classifiers $\left(\mathrm{e}_{\mathrm{i}} \mathrm{i}=\right.$ 1 to $\mathrm{J}$ ) are established on comparing an incoming $\mathrm{x}$ features with those of the reference models (prototypes) $\mathrm{x}_{\mathrm{i}}$ (where $\mathrm{i}=1$ to $\mathrm{N}$ or $\mathrm{n}$ according to the nature of problem). $\mathrm{N}$ represents total number of prototypes of $\mathrm{M}$ classes and $\mathrm{n}$ that of a single class. Euclidian distances $\mathrm{d}\left(\mathrm{x}, \mathrm{x}_{\mathrm{i}}\right)$ are computed. DempsterShafer evidence theory gives a decision concerning the class of the incoming pattern using the evidence masses. In case of a verification problem, two classes system (acceptance class $\mathrm{C} 1$ and rejection class $\mathrm{C} 2$ ) is created according to a threshold value. In this way, some $\mathrm{k}_{1}$ number of prototypes leads to $\mathrm{C} 1$ and $\mathrm{k}_{2}$ to $\mathrm{C} 2$ with respect to $\mathrm{n}$ models of the reference class such that $\mathrm{n}=\mathrm{k}_{1}+\mathrm{k}_{2}$. These threshold values are found during training phase. A single classifier itself takes a decision that $\mathrm{x} \in \mathrm{C} 1$ or $\mathrm{C} 2$. For a comparison purpose, a single global classifier based on whole features set is also studied.

\section{Signature recognition}

Handwritten signatures provide secure means for authentication. A handwritten signature based system has to solve variability problems and detection of forgeries (random, unskilled and skilled forgeries). While a large portion of the work is focused on random forgery detection, more efforts are still needed to address the problem of skilled forgery detection [4].

A lot of useful features have been used to differentiate the signatures. A work on efficient feature extraction system is still needed [1], [2], [3], [8], [9].

In our methodology, we are studying three types of signatures normalized histograms (horizontal, vertical and along siganture's principal axis), invariant toward either image resolution, or the signature size.

Other features used are some fractal features, global and local fractal dimension and mass dimension. The geometrical features employed are slant angle, classical contours and exterior contours. All of these features are described in detail in [10]. Besides our own signature dataset, a database was downloaded from a web site [11], containing data from 39 individuals: 24 genuine signatures for each individual, plus 30 forgeries. 


\section{Hand recognition}

Hand recognition systems are suggested to be very appropriate, because they do not cause anxiety for the user as fingerprint or iris systems do. It is possible to extract two types of biometric indicators from hand images; (i) hand geometry (ii) palmprint features. In conventional systems, pegs are used to fix the hand placement, but not in recent systems. Earlier efforts have used a set of various features but no individual results have been presented. As a by-product, our contribution presents this lacking comparison between different features.

Hand recognition systems are not new, there exist some patent works in 70's and also commercial systems [12]. All of the various methods proposed [13], [14], [15], [16], [17] use various geometric features (width, height and length of the fingers, hand size, height profile, etc.).

In our system, an ordinary scanner is used to acquire the hand image data. Examples of some acquired hand images using 80 dpi are shown in Figure 1. Otsu's method gives a binary image. Alignment is an important step in pegs free system.

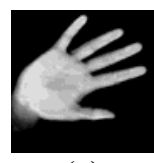

(a)

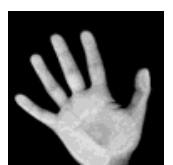

(b)

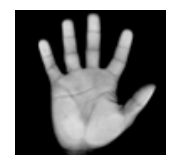

(c)
Figure 1: Scanned hand images of three individuals

We have studied a variety of features, to establish various classifiers. With localisation of different points as illustrated in Figure 2, it is possible to measure several important features of hand geometry. Fingers baselines, fingers lengths, fingers widths measurements at 5 fixed points, hand area, palm's width (segment $A B$ ), wrist's width (segment $C D$ ).

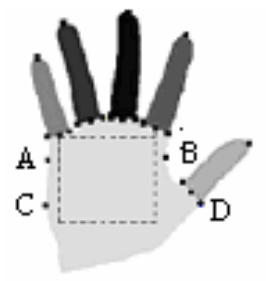

Figure 2: Localization of key points in hand image.

Palmprint area for hand lines detection is considered into a square shape as shown in the Figure 3. Sobel operator is used to extract the line information. Line features from the palmprint edge maps are detected using directional zones. Each of theses zones can detect lines oriented in 8 directions, i.e. east, west, north, south, north-east, south-east, south-west and north-west. Final direction of a zone corresponds to max score obtained in one of the possible directions.

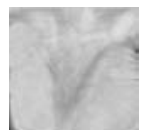

(a)

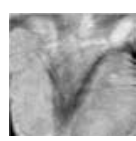

(b)

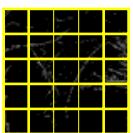

(C)
Figure 3. palmprint image (a) segmented, (b) enhanced image (c) Sobel palmprint lines in 25 squares.

\section{Experimental results}

In case of signature identification, initial data was collected from 35 persons who were asked to sign for 15 times. A leave one out method was used to evaluate the performances. Results for feature performance and combination approaches are shown in the tables.

Table 1: Performances for signature identification.

\begin{tabular}{|l|c|}
\hline Four classifiers and fusion approaches & Recognition \\
\hline Horizontal histogram (e1) & $78.8 \%$ \\
\hline Vertical histogram (e2) & $58.6 \%$ \\
\hline $\begin{array}{l}\text { Histogram along signature's principal } \\
\text { axis (e3) }\end{array}$ & $82.7 \%$ \\
\hline Fractal and geometrical features (e4) & $73.6 \%$ \\
\hline Bayesian approach (confusion matrix) & $96.6 \%$ \\
\hline D-S evidence theory & $96.9 \%$ \\
\hline Borda Count method & $96.3 \%$ \\
\hline $\begin{array}{l}\text { Majority vote rule } \\
\text { (i) with a threshold } \geq 0,5\end{array}$ \\
$\begin{array}{l}\text { (ii) with weighting of classifiers in } \\
\text { function of their performances }\end{array}$ & $88.8 \%$ \\
\hline Hidden Markov Models (HMM) & $99.6 \%$ \\
\hline
\end{tabular}

In case of signature verification we used a database available on Internet [11]. In [11] four SVM classifiers based on geometrical properties are studied. Their results are also shown in the table 2 .

Our simple distance classifier based on histograms was less performing than the other classifier based on fractal and geometrical features. To enhance further performance, we employed the majority vote rule, Borda count method to combine these classifiers. The performance was not remarkable as compared to that of the single averaging feature classifier (table 1). It can be noticed that with Dempster-Shafer theory refined results were obtained. The influence of parameter $\mathrm{k}$ itself has been studied with values from 3 to $n(n=12)$. From the experiments, the best results 
were found with $\mathrm{k}=8$, so the results shown in the table 1 for evidence theory are on this basis.

Table 2: Performances for signature verification (results are given as indicated in [11]).

\begin{tabular}{|l|c|}
\hline \multicolumn{1}{|c|}{ Classifiers / fusion approaches } & Equal Error Rate \\
\hline Contour measure [11] & $25.10 \%$ \\
\hline Contour following [11] & $25.94 \%$ \\
\hline Region grouping [11] & $25.83 \%$ \\
\hline Direct image [11] & $18.85 \%$ \\
\hline Histograms based classifier (e1) & $26.65 \%$ \\
\hline Fractal + geometrical features (e2) & $9.46 \%$ \\
\hline A single (e1+ e2) classifier & $7.35 \%$ \\
\hline Fusion by majority rule (e1, e2) & $7.19 \%$ \\
\hline Fusion by Evidence theory (e1, e2) & $5.88 \%$ \\
\hline
\end{tabular}

Hand identification and verification : Like previous application and in the same way we have first evaluated the performances of our four classifiers and then a fusion is realised. The results obtained are indicated in the tables 3 and 4 .

Table 3: Performances for hand identification.

\begin{tabular}{|l|c|}
\hline Four classifiers and fusion & Recognition \\
\hline Fingers lengths set (e1) & $92.3 \%$ \\
\hline Fingers widths set (e2) & $94.6 \%$ \\
\hline Fingers base lines set (e3) & $85.4 \%$ \\
\hline Rest of features set (e4) & $90.8 \%$ \\
\hline Majority vote rule (i) with a threshold & $93.9 \%$ \\
(ii) with weighting of classifiers & $96.1 \%$ \\
\hline Borda count method & $95.4 \%$ \\
\hline Bayesian approach (confusion matrix) & $96.9 \%$ \\
\hline Dempster-Shafer evidence theory & $96.9 \%$ \\
\hline
\end{tabular}

Table 4: Performances for hand verification.

\begin{tabular}{|l|c|c|}
\hline Four classifiers and fusion & FRR (\%) & FAR (\%) \\
\hline Finger lengths set (e1) & 2.2 & 1.8 \\
\hline Finger widths set (e2) & 2.2 & 2.0 \\
\hline Fingers base lines set (e3) & 2.2 & 2.2 \\
\hline Rest of features set (e4) & 3.6 & 3.1 \\
\hline A single (e1-e4) classifier & 1.5 & 0.6 \\
\hline $\begin{array}{l}\text { Fusion by majority vote (at } \\
\text { decision level) }\end{array}$ & 1.3 & 0.4 \\
\hline Fusion by D-S theory & 0.8 & 0.3 \\
\hline
\end{tabular}

\section{Conclusion and perspective}

We presented a fusion methodology based on Dempster-Shafer theory for two biometric applications. Different features according to each application were divided into various sets for classification with k-NN rule. Robustness of these classifiers was initially evaluated and their fusion with the help of Demsper-Shafer evidence theory increased the system performance in both applications as compared to voting majority rule, Borda count method, Bayesian approach, Hidden Markov models. In our future work, we intend to use other features and consequently influence of selection of features on overall system's performance will also be studied.

\section{References}

[1] R. Bajaj, S. Chaudhury, "Signature verification using multiple neural classifiers", Pattern Rec., 30 (1), 1997 1-7.

[2] H. Cardot, M. Revenu, "A static signature verification system based on a cooperating neural networks architecture", Pattern Recog. and Art. Intell. vol. 8 (3), 1994, 679-692.

[3] X. Xiao, G. Leedham, "Signature verification using a modified Bayesian network", Pat., Rec., 35, 2002, 983-995.

[4] E.R.J. Justino, F. Bortolozzi, R. Sabourin, "Off-line signature verification using HMM for random, simple and skilled forgeries", Proc. of the $6^{\text {th }}$ ICDAR, 2001, 1031-1034.

[5] C. Allgrove, M.C. Fairhurst, "Majority voting for improved signature verification" IEE Colloquium on Visual Biometrics, London, 2000 (Ref No. 2000/018), pp. 9/1-9/4.

[6] G. Shafer, A mathematical Theory of Evidence, Princeton Univ. Press, Princeton New Jersey, 1976.

[7] M. Arif, T. Brouard, N. Vincent, "Non parametric fuzzy modeling of belief function in evidence theory", $15^{\text {th }}$ Conf of IASTED, MS2004, March 1-3, Marina Del Rey CA. 60-65.

[8] B. Fang, C.H. Leung, Y.Y. Tang, K.W. Tse, P.C.K. Kwok, "Off-line signature verification by the tracking of feature and stroke positions", Pat. Rec., 36, 2003, 91-101.

[9] R. Sabourin, G. Genest and F. Preteux , "Offline signature verification by local granulometric size distributions", IEEE Trans. PAMI 19 (8), 1997, 976-988.

[10] M. Arif, T. Brouard, N. Vincent, "A fusion methodology for recognition of off-line signatures", The $4^{\text {th }}$ PRIS-2004, 13-14 April, Porto, Portugal, pp. 35-44.

[11] http://www.gpds.ulpgc.es/download/index.htm.

[12] HaSIS, www.csr.unibo.it/ research/Biolab/hand.htm.

[13] A. K. Jain, A. Ross, S. Pankanti, "A prototype hand geometry-based verification system," In Proc 2nd Int. Conf. AVBPA, Washington, DC, USA. 1999, 166-171.

[14] A. Kumar, D.C.M. Wong, H.C. Shen, A.K. Jain. "Personal verification using palmprint and hand geometry biometric". Springer-Verlag GmbH 2688 / 2003. 668-678.

[15] C.M. Travieso, J.B. Alonso, S. David, M.A. Ferrer, "Optimization of a biometric system identification by hand geometry" CSIMTA'2004, Cherbourg, France, 581-586.

[16] R. Sanchez-Reillo, C. Sanches-Avila, and A. GonzalesMarcos, "Biometric identification through hand geometry measurements," IEEE Trans. Pattern Anal. \& Mach. Intell. 22 (10), 2000, 1168-1171.

[17] C. Oden, A. Ercil, and B. Buke, "Combining implicit polynomials and geometric features for hand recognition," Pattern Recognition letters, 24(13), 2003, 2145-2152. 